\title{
2-SR-Based Electrically Small Antenna for RFID Applications
}

\author{
Ferran Paredes ${ }^{1}$, Simone Zuffanelli ${ }^{1}$, Pau Aguilà ${ }^{1}$, \\ Gerard Zamora $^{1}$, Ferran Martin ${ }^{1}$, and Jordi Bonache ${ }^{1}$ \\ ${ }^{1}$ Department of Electronic Engineering, Universitat Autònoma de Barcelona, Barcelona, Spain \\ *corresponding author, E-mail: Ferran.paredes@uab.es
}

\begin{abstract}
In this work, the 2-turns Spiral Resonator (2-SR) is proposed as an electrically small antenna for passive radio frequency identification (RFID) tags at the European UltraHigh Frequency (UHF) band. The radiation properties are studied in order to explore the viability of the 2-SR applied to tag antenna design. Based on analytical calculations, the radiation pattern is found to provide a cancellation of the radiation nulls. This results in a mitigation of the blind spots in the read range, which are present in typical UHF-RFID tags as an undesired feature. As a proof of concept, a passive tag of size $35 \mathrm{~mm} \times 40 \mathrm{~mm}\left(\lambda_{0} / 10 \times \lambda_{0} / 9\right)$ based on the 2-SR antenna is designed and fabricated. Good radiation efficiency (75\%) and a quasi-isotropic radiation pattern are obtained. The experimental tag read range for different directions is in good agreement with the simulation results. The measured read range exhibits maximum and minimum values of $6.7 \mathrm{~m}$ and $3.5 \mathrm{~m}$, respectively.
\end{abstract}

\section{Introduction}

Radio frequency identification (RFID) is a technology that provides objects tagging and tracking capability by means of electromagnetic waves [1]. RFID systems can operate at low frequencies (LF) and high frequencies (HF), although normally the most convenient band is the ultra-high frequency (UHF) since UHF-RFID systems can provide larger memory, faster reading, more secure data transmission and superior read range [1]. Such a read range can be still enhanced by using active tags, since they include a battery. However, passive tags are more attractive due to low cost and small dimensions.

UHF-RFID systems are composed by readers and tags. Readers send an electromagnetic query signal to the tags, which generate a modulated backscattered wave as a response. Then, readers receive such a backscattered signal and identify the tagged objects. Passive UHF-RFID tags consist of an antenna matched to an application specific integrated circuit (ASIC), usually by means of a T-match network [2,3]. RFID tag antennas are typically based on electric half-wave dipoles, which are meandered [4] in order to shrink tag dimensions, since size reduction is one of the most challenging aspects in RFID.

Spiral resonators are commonly used in planar microwave devices due to its small size, which can be further reduced by increasing the number of turns. The 2- turns spiral resonator (2-SR) is an example of spiral, which has been recently applied to the design of chipless RFID tags [5], and it has been also used to design metamaterial devices at microwave frequencies [6]. Metamaterial-inspired resonators, e.g. the split ring resonator (SRR) or its complementary counterpart (CSRR) [7], the open split ring resonators (OSRR) [8], among others, have been applied to electrically small antenna design $[9,10]$, since their physical dimensions are much smaller than the wavelength at the working frequency. Specifically, this feature has been also employed to develop size optimized RFID tags [11]. Moreover, spirals have been also used to achieve dual band behavior in RFID tags [12] .

In this work, the radiation properties of the 2-SR are analytically studied. It is known that the 2-SR behavior at the first (fundamental) resonance is similar to that of a small loop antenna [6]. However, the proposed analysis is focused on the radiation characteristics beyond the first resonance, where the antenna is expected to provide proper impedance matching with the ASIC. Within the explored frequency range, the 2-SR is still electrically small and, therefore, its radiation properties are mainly determined by its first-order (dipole) electric and magnetic moments. By taking advantage of the bi-anisotropy of the particle, a quasiisotropic radiation pattern is obtained by controlling both the electric and magnetic dipole moments. This can be useful to mitigate the blind spots in the reading patterns of UHFRFID tags, which are present in most of conventional tags as an undesired feature. Based on the results, a small quasiisotropic passive tag working at the European UHF-RFID band (865.6 - 867.6 MHz) [13] is developed.

The paper is organized as follows. At Section 2 the analysis of the 2-SR antenna is presented. The validation of such analysis by means of electromagnetic simulation is carried out at section 3. The design of a passive UHF-RFID tag is proposed and developed at Section 4, and the experimental results, in terms of read range, are described in Section 5. Finally, conclusions are explained at Section 6.

\section{Analysis of the 2-SR radiation properties}

The topology of the 2-SR is depicted in Figure 1a. In order to analyze the structure, the resonator is divided into two rings, the internal $A$ (with radius $r_{A}$ ) and external $B$ (with radius $r_{B}$ ). The mean radius of the 2-SR is defined as $r_{0}=\left(r_{A}+r_{B}\right) / 2$. The geometric parameters $c, d$ and $\varphi$ 

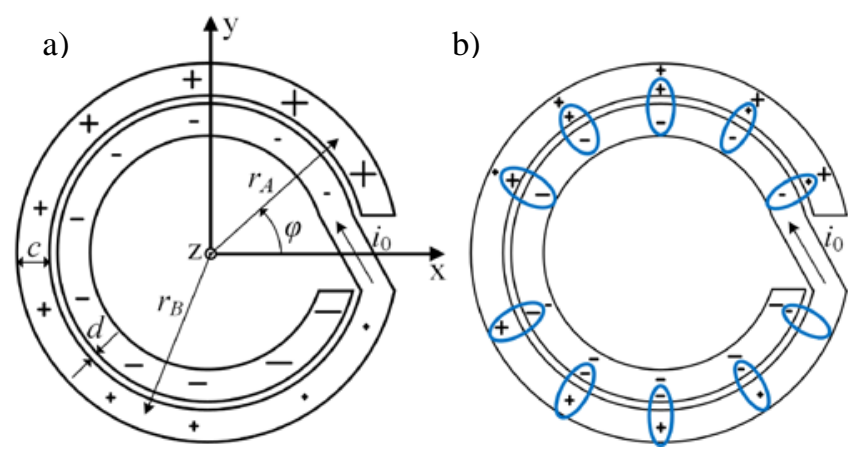

Figure 1: (a) Topology and electric charge distribution of the 2-SR at the operation frequency $f_{0}$ and (b) decomposition of the electric charge distribution in the upper half of the external ring and in the lower half of the internal ring. The resulting electric dipole moments in the radial direction are also shown.

represent the rings width, the distance between the rings and the angular position with respect to the $x$-axis, respectively.

Let us now proceed to analyze the current inside the 2$\mathrm{SR}$. At the fundamental resonant frequency $f_{1}$ the particle is electrically small with a radius of about $1 / 40$ wavelengths. Therefore, the current distribution is well approximated by a triangular function, where the maximum current amplitude $i_{0}$ occurs at the geometrical center of the 2-SR, and the current on each ring vanishes at the cuts. However, when the operation frequency $f_{0}$ is higher than the 2-SR fundamental resonance $f_{1}$, the current along the particle is assumed to be sinusoidal. Let us consider the case in which the total length of the particle is roughly half-wavelength at $f_{0}$. Under this assumption, the current flows in the same direction on both rings and it exhibits a maximum at the 2-SR geometrical center (approximately at $\varphi \approx 2 \pi$ for the external ring and $\varphi \approx 0$ for the internal ring). Such a current distribution can be written as

$$
\begin{aligned}
& \boldsymbol{i}_{A}(\varphi, t)=i_{0} \sin (\varphi / 4) \cos \left(\omega_{0} t\right) \hat{\boldsymbol{\varphi}}, \\
& \boldsymbol{i}_{B}(\varphi, t)=i_{0} \cos (\varphi / 4) \cos \left(\omega_{0} t\right) \hat{\boldsymbol{\varphi}},
\end{aligned}
$$

where $\varphi \in[0,2 \pi]$, and $\omega_{0}$ is the working angular frequency. The magnetic dipole moment associated to the 2-SR external ring can be calculated as

$$
\boldsymbol{m}_{A}=\frac{1}{2} \int_{0}^{2 \pi} \hat{\boldsymbol{r}} \times \mathbf{i}_{A}(\varphi, t) r_{A}^{2} d \varphi,
$$

obtaining the following expression

$$
\boldsymbol{m}_{A}=2 r_{A}^{2} i_{0} \cos \left(\omega_{0} t\right) \hat{\mathbf{z}} .
$$

In the same way, the magnetic dipole moment for the internal ring can be calculated as

$$
\boldsymbol{m}_{B}=2 r_{B}^{2} i_{0} \cos \left(\omega_{0} t\right) \hat{\mathbf{z}} .
$$

The total magnetic dipole moment is the sum of both moments, which can be related to the mean ring radius $r_{0}$, approximately by

$$
\boldsymbol{m} \approx 4 r_{0}^{2} i_{0} \cos \left(\omega_{0} t\right) \hat{\mathbf{z}} .
$$

The approximation considered in (6) assumes that the internal and external rings have similar radii, which is

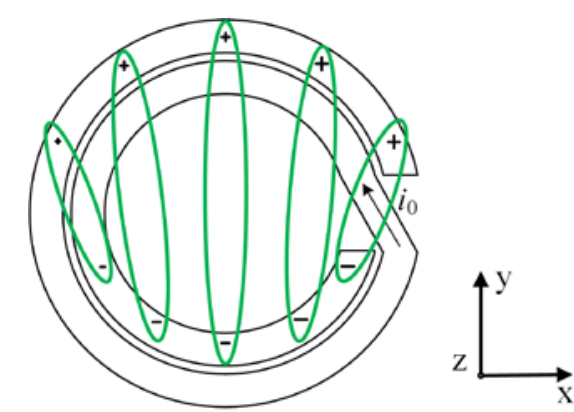

Figure 2: Residual electric charge distribution from the decomposition of Figure $1 \mathrm{~b}$ that mainly provides electric dipole moments along the $y$-direction.

typically satisfied. The radiated power associated to a magnetic dipole moment of amplitude $m_{0}$ can be written as [14]

$$
P_{\text {rad }}^{M}=\frac{Z_{0} k^{4} m_{0}^{2}}{12 \pi},
$$

where $Z_{0}$ is the free-space impedance and $k$ is the free-space wavenumber. It follows that the radiation resistance referred to the maximum current amplitude $i_{0}$ is given by

$$
R_{\mathrm{rad}}^{M}=\frac{128}{3} Z_{0} \pi^{3}\left(\frac{r_{0}}{\lambda_{0}}\right)^{4} \approx 5 \cdot 10^{5}\left(\frac{r_{0}}{\lambda_{0}}\right)^{4},
$$

where $\lambda_{0}$ is the free-space wavelength at the operation frequency $f_{0}$.

In order to obtain the radiation resistance provided by the electric dipole moment associated to the 2-SR, the linear charge density for each ring is found by solving the electric charge continuity equation from (1) and (2), obtaining

$$
\begin{aligned}
& \lambda_{A}(\phi, t)=\frac{1}{4 r_{A} \omega_{0}} i_{0} \cos (\varphi / 4) \sin \left(\omega_{0} t\right), \\
& \lambda_{B}(\phi, t)=\frac{-1}{4 r_{B} \omega_{0}} i_{0} \sin (\varphi / 4) \sin \left(\omega_{0} t\right) .
\end{aligned}
$$

According to (9) and (10), the charge density vanishes at the transition between rings, and exhibits a maximum at the 2SR edges (see Figure 1a). In order to simplify the calculation of the electric dipole moment, the charge density was decomposed into the sum of two charge densities in the upper half of the external ring, i.e. $\varphi \in[0, \pi]$, and in the lower half of the internal ring, i.e. $\varphi \in[\pi, 2 \pi]$, according to Figure 1b. The first term is composed by the charges that form electric dipole moments in the radial direction between the internal and external rings (see Figure 1b). The rest of the charges mainly provide an electric dipole moment oriented along the $y$-direction between the upper half of the external ring and lower half of the internal ring (see Figure 2). There is also a slight contribution of the electric dipole moment along the $x$-direction (see Figure 2) which can be neglected provided that $r_{\mathrm{A}} \approx r_{\mathrm{B}}$, a condition usually satisfied.

Concerning the electric dipole moments in the radial direction, the contributions of the upper and lower halves of the 2-SR result in a cancellation in the $y$-direction. On the other hand, a constructive contribution in the $x$-direction arises, and can be calculated as 


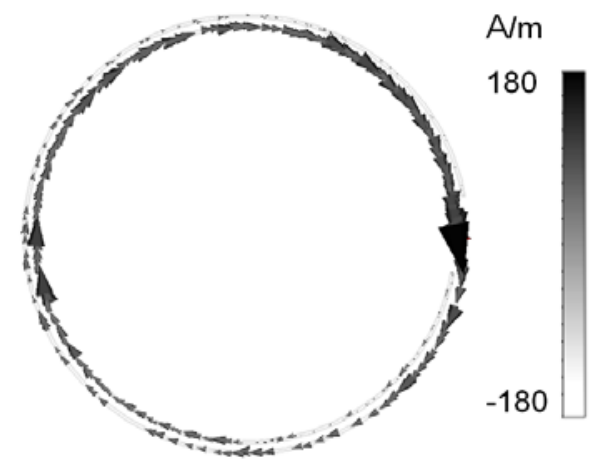

Figure 3: Electric current density distribution of the simulated 2-SR without considering any substrate, nor ohmic losses.

$$
p_{x}=2 \int_{0}^{\pi}-\lambda_{B}(\varphi, t)(c+d) r_{B} \cos (\varphi) d \varphi .
$$

By integrating (11), we obtain

$$
p_{x}=-\frac{(2+\sqrt{2})}{15} \frac{(c+d)}{\omega_{0}} i_{0} \sin \left(\omega_{0} t\right) .
$$

The total electric dipole moment along the $y$-direction obtained from the charge distribution depicted in Figure 2, can be calculated as

$$
p_{y}=\int_{0}^{\pi} \lambda_{\text {eff }}(\varphi, t) 2 r_{0}^{2} \sin (\varphi) d \varphi
$$

where $\lambda_{\text {eff }}=\lambda_{\mathrm{A}}+\lambda_{\mathrm{B}}$ is the effective linear charge density, and corresponds to the residual charges after considering the abovementioned set of radial electric dipole moments. Integration of (13) gives

$$
p_{y}=\frac{8}{15} \frac{r_{0}}{\omega_{0}} i_{0} \sin \left(\omega_{0} t\right) \text {. }
$$

A comparison between (12) and (14) reveals that the electric dipole moment is much higher along the $y$-direction than the $x$-direction, since $r_{0}>>(c+d)$. Therefore, the total electric dipole moment can be approximated by

$$
\boldsymbol{p} \approx p_{y} \hat{\boldsymbol{y}} .
$$

Thus, it is possible to calculate the radiated power, neglecting the contribution along the $x$-direction, by using the Larmor equation [15], given by

$$
P_{\text {rad }}^{E}=c_{0}^{2} \frac{Z_{0} k^{4} p_{0}^{2}}{12 \pi},
$$

where $c_{0}$ is the speed of light in vacuum and $p_{0}$ is the amplitude corresponding to the electric dipole moment (15). It follows that the radiation resistance referred to the maximum current amplitude $i_{0}$ can be approximated by

$$
R_{\mathrm{rad}}^{E}=\frac{128}{675} \pi Z_{0}\left(\frac{r_{0}}{\lambda_{0}}\right)^{2} \approx 224\left(\frac{r_{0}}{\lambda_{0}}\right)^{2} .
$$

Since the electric and the magnetic dipole moments are in phase quadrature [as can be deduced by comparing (6) and (15)], they behave as orthogonal radiators. Therefore, the total radiation resistance $R_{\text {rad }}$ of the particle can be evaluated as the sum of the radiation resistances related to the magnetic (8) and the electric (17) dipole moments.
As mentioned before, the radiated fields of a 2-SR working at a frequency region, where the particle is electrically small, can be predicted from the generated first order moments. In such a region, they can be considered elemental radiators to a good approximation (as it has been done in the analysis above). It can be seen by comparing (6) and (15) that a $90^{\circ}$ angle exists between the electric and magnetic dipole moments, which are oriented along the $y$ axis and the $z$-axis, respectively. Moreover, as said before, a $90^{\circ}$ phase-shift between the two radiators exists. As a result, a bi-directional radiation pattern is expected to occur with a maximum directivity of $1.5(1.76 \mathrm{~dB})$ [16]. The directions where the fields radiated by the magnetic and electric moments are orthogonal corresponds to the $\varphi=90^{\circ}$ plane, in which the radiation of the electric moment is purely crosspolar. Since the electric dipole moment is oriented along the $y$-axis, its radiation is maximized at $\theta=\left\{0^{\circ}, 180^{\circ}\right\}$. Hence, in these directions, the value of the cross-polarization level is maximized. Its value (normalized to the maximum copolar radiated power) can be found by assuming that the radiation pattern of both moments present the same shape (i.e. elemental radiators), so that it can be directly evaluated by dividing (17) by (8), obtaining

$$
\mathrm{XPOL}_{\max }=\frac{R_{\mathrm{rad}}^{E}}{R_{\mathrm{rad}}^{M}}=\frac{3}{675 \pi^{2}}\left(\frac{r_{0}}{\lambda_{0}}\right)^{-2} \approx \frac{1}{2220}\left(\frac{r_{0}}{\lambda_{0}}\right)^{-2} .
$$

As it will be detailed in the next section, this relation is useful to evaluate the read range of the tag in the $z$ direction, normalized to the tag maximum read range $(x-$ direction). In fact, instead of presenting a null read range along the $z$-axis, where the magnetic radiation vanishes, the tag is expected to show a residual read range produced by the electric radiation.

\section{Analysis validation}

To validate the radiation properties analyzed in Section II, a 2-SR was designed and simulated by means of the commercial software CST Studio Suite. The electromagnetic simulation was performed without considering any substrate, nor ohmic losses. The structure was excited through a differential port connected across a cut $(0.2 \mathrm{~mm})$ opened in the 2-SR at the ring center, where a current maximum $i_{0}$ is expected. The values for the geometric parameters of the particle are $r_{A}=13.65 \mathrm{~mm}, r_{B}=14.35 \mathrm{~mm}, c=0.2 \mathrm{~mm}$, $d=0.5 \mathrm{~mm}$ and the cut width was set to $5 \mathrm{~mm}$. The mean ring radius $r_{0}=14 \mathrm{~mm}$ (1/25 wavelengths) was chosen so that the 2-SR total length is approximately $\lambda_{0} / 2$ at the intermediate frequency of the European UHF-RFID band, $f_{0}=867 \mathrm{MHz}$. This feature and the fact that the widths of the rings are forced to be very small in terms of wavelength, allows considering the sinusoidal current distribution corresponding to (9) and (10) on the 2-SR as a good approximation.

From the previous analysis, the radiation resistance associated to the magnetic dipole moment (8) and to the electric dipole moment (17) at the operation frequency $f_{0}$ can be predicted, resulting in $1.34 \Omega$ and $0.37 \Omega$, respectively. 


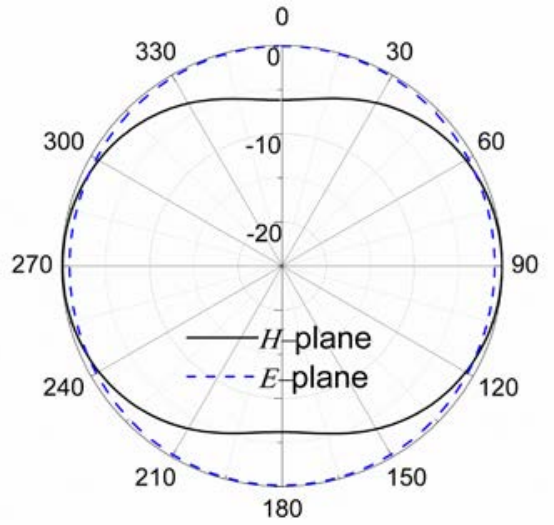

Figure 4: Simulated normalized radiation pattern of the 2SR at $f_{0}$ for the $H$-plane $\left(\varphi=0^{\circ}\right)$ and $E$-plane $\left(\theta=90^{\circ}\right)$.

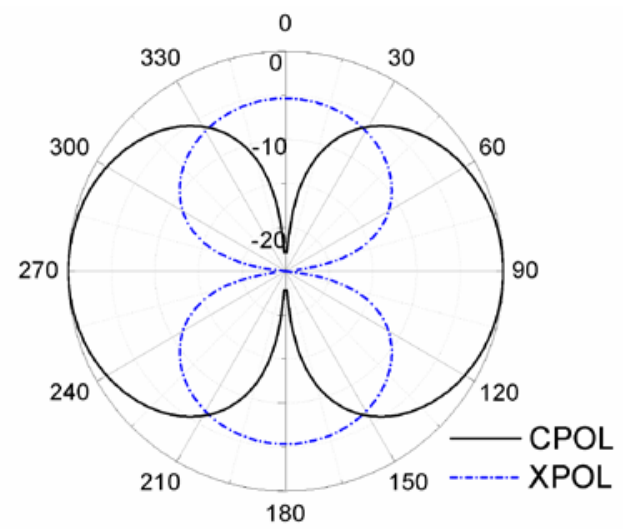

Figure 5: Simulated copolar (associated to the magnetic dipole moment) and cross-polar (associated to the electric dipole moment) components of the 2-SR at $f_{0}$ for the $y z$ plane $\left(\varphi=90^{\circ}\right)$.

Thus, the total radiation resistance is expected to be $R_{\text {rad }}=1.71 \Omega$, and the theoretical maximum cross-polar component (18) gives XPOL $_{\max }=-5.6 \mathrm{~dB}$.

The results obtained by simulation show an input impedance $Z_{\text {in }}=1.7+\mathrm{j} 398 \Omega$ at $f_{0}$. Perfect agreement between the simulated and analytical radiation resistance is achieved. Moreover, the assumption of a sinusoidal electric current distribution along the particle results in a good approximation, as can be seen in Figure 3, where the simulated current density distribution at $f_{0}$ is depicted. As expected, the maximum amplitude $i_{0}$ occurs at the geometrical center of the particle, where the port was positioned. A simulation of the normalized radiation pattern for the $E$-plane $\left(\theta=90^{\circ}\right)$ and $H$-plane $\left(\varphi=0^{\circ}\right)$ is shown in Figure 4. As expected, a bi-directional radiation pattern is obtained in the $E$-plane with a directivity $D_{0}=1.9 \mathrm{~dB}$ along the $x$-direction or, alternatively, $\varphi=\left\{0^{\circ}, 180^{\circ}\right\}$, which is similar to the theoretical value $(1.76 \mathrm{~dB})$. It can be appreciated in the $H$-plane that a residual radiation exists at $\theta=0^{\circ}$ (where the radiation of the magnetic dipole moment vanishes) due to the contribution of the electric dipole moment. Such a contribution can be quantified by analyzing the $\varphi=90^{\circ}$ plane (Figure 5), where it can be seen that
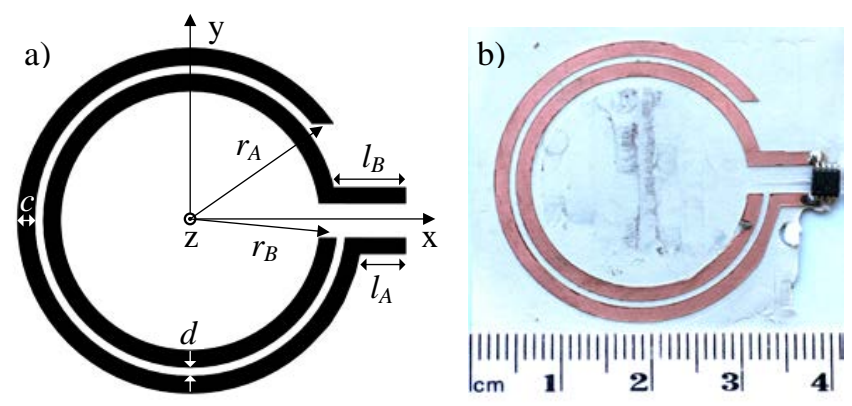

Figure 6: (a) Layout of the designed 2-SR antenna and (b) photograph of the fabricated tag prototype. The final dimension are $r_{A}=15.4 \mathrm{~mm}, r_{B}=13 \mathrm{~mm}, l_{A}=4.3 \mathrm{~mm}$, $l_{B}=6.8 \mathrm{~mm}, c=1.6 \mathrm{~mm}, d=0.8 \mathrm{~mm}$.

$\mathrm{XPOL}_{\max }=-5.2 \mathrm{~dB}$. Very good agreement with the analytical value $(-5.6 \mathrm{~dB})$ is achieved.

\section{Design of a 2-SR-based UHF-RFID tag}

Let us consider a typical UHF-RFID integrated circuit (the NXP SL3S1001FTT chip) for the design of a 2-SR-based tag. The input impedance of this integrated circuit at the operating frequency $f_{0}$ provided by the manufacturer is $Z_{c}=20.5-j 500 \Omega$. This value suggests that the 2-SR antenna can be tailored to be directly matched to the chip, since it presents a small resistance with a high inductive reactance. The proposed tag was designed on a commercial low loss microwave substrate, the Rogers RO3010 substrate with dielectric constant $\varepsilon_{r}=10.2$, loss tangent $\tan \delta=0.0023$ and thickness $h=0.254 \mathrm{~mm}$. The final layout is shown in Figure 6a. The geometrical dimensions of the tag antenna are $r_{A}=15.4 \mathrm{~mm}, r_{B}=13 \mathrm{~mm}, c=1.6 \mathrm{~mm}, d=0.8 \mathrm{~mm}$, $l_{A}=4.3 \mathrm{~mm}$, and $l_{B}=6.8 \mathrm{~mm}$. The width of the ring was increased with respect to the design presented in Section 3, in order to enhance the radiation efficiency of the 2-SR antenna. It also allows obtaining a higher value of the input resistance, in part, due to the proximity effect [17], which tends to separate the current densities between the internal and external rings, increasing the magnetic and electric radiation resistances.

The simulated input impedance was found to be $Z_{\text {in }}=5.7+\mathrm{j} 500 \Omega$ at $f_{0}$, which corresponds to a power reflection coefficient of $|s|=-5 \mathrm{~dB}$ at the tag resonance frequency. The simulated radiation efficiency, directivity and gains along the $x, y, z$-directions were found to be $\eta_{\text {rad }}=75 \%, D_{0}=1.85 \mathrm{~dB}, G_{x}=0.6 \mathrm{~dB}, G_{y}=-0.8 \mathrm{~dB}$ and $G_{z}=-3.6 \mathrm{~dB}$, respectively. The simulated radiation patterns in the $E$-plane $\left(\theta=90^{\circ}\right)$ and $H$-plane $\left(\varphi=0^{\circ}\right)$ are depicted in Figure 7 , where a bi-directional radiation pattern can be appreciated. The simulated tag exhibits a normalized crosspolar component of $\mathrm{XPOL}_{\max }=-2.8 \mathrm{~dB}$, as can be deduced from the $\varphi=90^{\circ}$ plane (see Figure 8), where the radiation of the electric moment is purely cross-polar. This provides an advantage in the tag read range, which can be inferred as [18] 


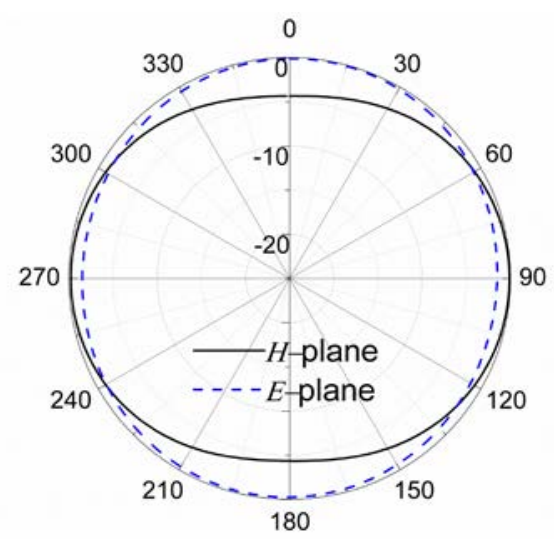

Figure 7: Simulated normalized radiation pattern of the designed 2-SR antenna, depicted in Figure 6a, at $f_{0}$ for the $H$-plane $\left(\varphi=0^{\circ}\right)$ and $E$-plane $\left(\theta=90^{\circ}\right)$.

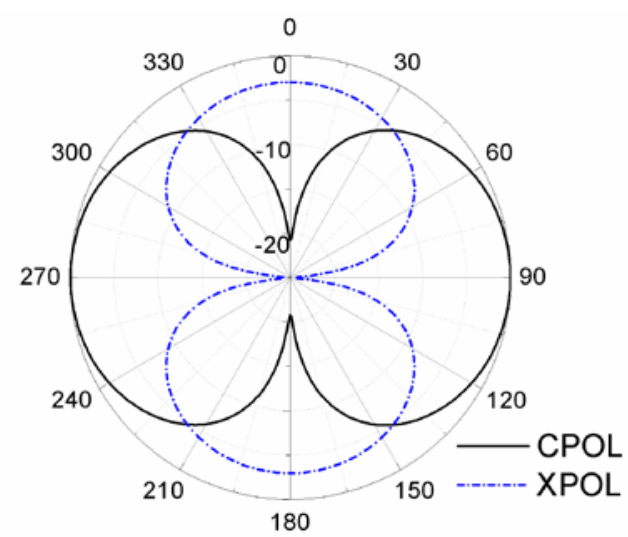

Figure 8: Simulated copolar (associated to the magnetic dipole moment) and cross-polar (associated to the electric dipole moment) components of the designed 2-SR antenna, depicted in Figure 6a, at $f_{0}$ for the $y z$-plane $\left(\varphi=90^{\circ}\right)$.

$$
R R=\frac{\lambda_{0}}{4 \pi} \sqrt{\frac{E I R P \cdot G_{r} \cdot \tau}{P_{\text {chip }}}},
$$

being $P_{\text {chip }}$ the minimum threshold power necessary to activate the RFID chip (the typical value for the NXP SL3S1001FTT chip is $-14 \mathrm{dBm}), G_{r}$ the gain of the tag antenna, $\tau$ the power transmission coefficient and EIRP the equivalent isotropically radiated power, determined by local country regulations (e.g. 3.3W in Europe and $4 \mathrm{~W}$ in USA). The main advantage of the proposed tag is that, unlike conventional tags, it exhibits a quasi-isotropic radiation pattern, which provides a mitigation of the blind spots in the tag read range. As said before, the value of $\mathrm{XPOL}_{\max }$ directly relates the read range in the minimum direction ( $z$ axis) to the read range in the maximum direction ( $x$-axis). Based on (19), it can be found that

$$
\frac{R R_{z}}{R R_{x}}=\sqrt{\frac{\mathrm{XPOL}_{\text {max }}}{1+\mathrm{XPOL}_{\text {max }}} .}
$$

The theoretical $R R$ for different directions (Figure 9), obtained on the basis of the simulated antenna gains and the

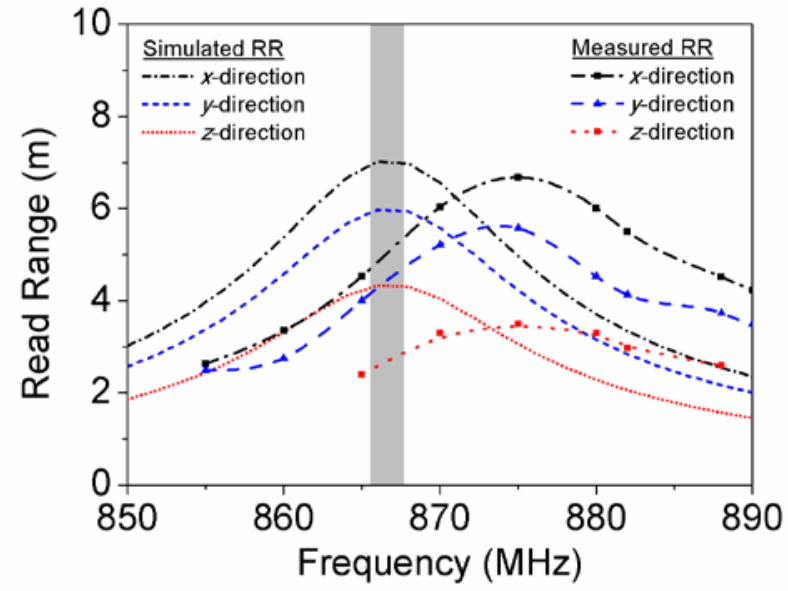

Figure 9: Simulated and measured read range for different 2SR tag orientations. The European frequency range (865.6 867.6 MHz) is depicted in grey.

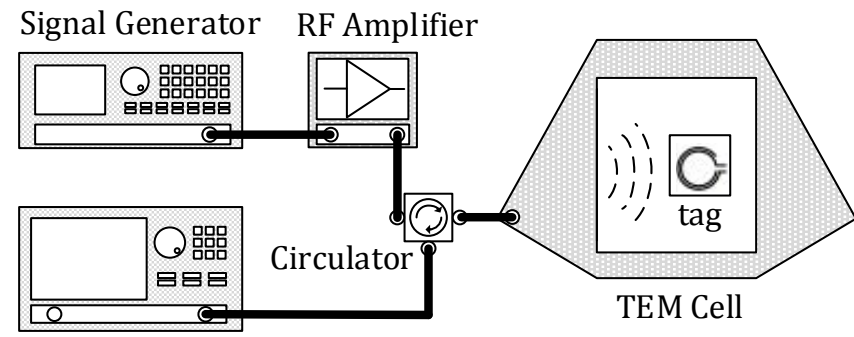

Signal Analyzer

Figure 10: Sketch of the RFID tags measurement setup.

power reflection coefficient, confirms the absence of blind spots. Moreover, it is in good agreement with the behavior predicted by (20), which estimates the theoretical ratio between minimum and maximum read ranges of 0.6 (taking into account that the simulated value of $\mathrm{XPOL}_{\max }$ is $-2.8 \mathrm{~dB})$.

\section{Experimental results}

Finally, the layout of the 2-SR antenna described in the previous section was fabricated by means of a LPKF-H100 drilling machine. The final dimensions are $35 \times 40 \mathrm{~mm}$ $\left(\lambda_{0} / 10 \times \lambda_{0} / 9\right)$. The ASIC was soldered to the 2 -SR antenna as it is shown in the prototype photography (see Figure 6b).

The RFID setup depicted in Figure 10 consists of an Agilent N5182A vector signal generator, which generates the RFID interrogation frames, connected to an RFPA $R F 101000-10$ solid state RF amplifier by means of a $50 \Omega$ coaxial cable. Such amplifier is connected to the TEM cell (Wavecontrol WaveCell) through a circulator. The prototype under test, in this case the 2-SR-based RFID tag, is located inside the TEM cell with the suitable orientation. When the tag is properly excited by the RFID frame, it sends a backscattered signal to the Agilent N9020A signal analyzer, which is in charge of decoding the RFID frames, through the circulator. To measure the read range, a sweep frequency by means of the vector signal generator is carried out and, at 
each frequency, the minimum power transmitted from the generator, $P_{\min }$, required to communicate with the tag is recorded. An electric probe (Wavecontrol EFCube) is placed into the TEM cell to determine the root mean square of the incident electric field $E_{\text {rms }}$, corresponding to the minimum power $P_{\min }$ at each frequency. To obtain the measured read range, the average power density $S$ associated to a plane wave, determined from the value of the electric field $E_{\text {rms }}$, is used according to

$$
S=\frac{E_{\mathrm{rms}}^{2}}{Z_{0}} .
$$

Moreover, the average power density can be also related to a transmitting antenna (e.g. reader antenna) according to

$$
S=\frac{P_{t} G_{t}}{4 \pi r^{2}}=\frac{E I R P}{4 \pi r^{2}}
$$

where $r$ is the distance from the antenna, $P_{t}$ and $G_{t}$ are the total transmission power and the transmission antenna gain, respectively. Then, by equating (21) and (22), the measured read range can be obtained as

$$
R R=\frac{\sqrt{30 E I R P}}{E_{\mathrm{rms}}} .
$$

The experimental read range was measured for different tag orientations and compared to the theoretical read range, as it can be seen in Figure 9. The measured maximum read range $R R_{x}=5.7 \mathrm{~m}$ is obtained along the $x$-direction, whereas the read ranges measured for a standalone magnetic and electric dipole moment contribution are $R R_{y}=4.9 \mathrm{~m}$ and $R R_{\mathrm{z}}=3.4 \mathrm{~m}$, respectively. Good agreement between simulated and measured results is achieved. However, a $10 \mathrm{MHz}$ frequency shift can be observed between measurement and simulation. This is attributed to a slight shift in the power reflection coefficient towards high frequencies, which can be due to fabrication tolerances and variations on the ASIC impedance.

\section{Conclusion}

In this paper, the radiation properties of the 2-SR has been analyzed in terms of radiation resistance, maximum crosspolar component and radiation pattern. The study concludes that the 2-SR has associated an electric and magnetic dipole moments, which involves a bi-directional radiation pattern. Consequently, the blind spots, which are present when using elemental radiators, have been mitigated. By taking advantage of this feature, a passive UHF-RFID tag based on quasi-isotropic 2-SR antenna with a high radiation efficiency has been designed and fabricated. The experimental read range, measured along different directions, reveals the absence of blind spots with maximum and minimum values of $6.7 \mathrm{~m}$ and $3.5 \mathrm{~m}$, respectively. Good agreement between simulation and experimental results are achieved. The results reveal that the 2-SR RFID tag is competitive considering its electrically small size and its radiation characteristics.

\section{Acknowledgements}

This work has been supported by MINECO-Spain (projects TEC2013-40600-R COM-SEN-RFID), by FEDER funds, and by Generalitat de Catalunya (project 2014SGR-157). Ferran Martín is in debt to ICREA for funding his work.

\section{References}

[1] K. Finkenzeller, RFID handbook: Radio-Frequency Identification fundamentals and applications, 2nd ed: John Wiley and Sons, 2003.

[2] G. Zamora, S. Zuffanelli, F. Paredes, F. Martin, and J. Bonache, Design and synthesis methodology for UHFRFID tags based on the t-match network, IEEE Trans. Microw. Theory Tech., vol. 61, no. 12, pp. 4090-4098, 2013.

[3] N. A. Mohamed, K. R. Demarest, and D. D. Deavours, Analysis and synthesis of UHF RFID antennas using the embedded T-match, Proc. IEEE Int. Conf. RFID, Apr. 2010, pp. 230-236.

[4] G. Marrocco, Gain-optimized self-resonant meander line antennas for RFID applications, Antennas Wireless Propag. Lett., vol. 2, no. 21, pp. 302-305, 2003.

[5] Preradovic S., N. Kamakar, E.M. Emran, Chipless RFID tag with integrates resistive and capacitive sensors, Proc. of Asia-Pacific Microw. Conf. (APMC), Melbourne, Australia, December 2011, 1354-1357.

[6] R. Marques, F. Martin, M. Sorolla, Metamaterials with Negative Parameters: Theory, Design and Microwave Applications, John Wiley and Sons, 2008.

[7] J. D. Baena, J. Bonache, F. Martin, R. M. Sillero, F. Falcone, T. Lopetegi, M. A. G. Laso, J. García-García, I. Gil, M. F. Portillo, and M. Sorolla, Equivalent-circuit models for split-ring resonators and complementary split-ring resonators coupled to planar transmission lines, IEEE Trans. Microw. Theory Tech., vol. 53, no. 4, pp. 1451-1461, 2005.

[8] J. Martel, R. Marqués, F. Falcone, J. D. Baena, F. Medina, F. Martín, and M. Sorolla, A new LC series element for compact band pass filter design, IEEE Microw. Wireless Comp. Lett., vol. 14, no. 5, pp. 210 212, May 2004.

[9] K. B. Alici, E. Ozbay, Electrically small split ring resonator antennas, J. Appl. Phys., vol. 101, no. 8, paper 083104, 2007.

[10] J.J. Ma, X.Y. Cao, T. Liu, Design the size reduction patch antenna based on complementary split ring resonators, IEEE Int. Conf. Microw. Millim. Wave Tech., Chengdu, pp. 401-402, May 2010.

[11]B. D. Braaten, R. P. Scheeler, M. Reich, R. M. Nelson, C. Bauer-Reich, J. Glower and G.J. Owen, Compact metamaterial-based UHF RFID antennas: deformed omega and split-ring resonator structures, ACES Special Issue on Comput. Experimental Tech. RFID Syst. Applicat., vol. 25, no. 6, 2010.

[12] F. Paredes, G. Zamora, F. J. H. Martinez, F. Martin, J. Bonache, Dual-band UHF-RFID tags based on meander-line antennas loaded with spiral resonators, 
IEEE Antennas Wireless Propag. Lett., vol. 10, pp. 768771, 2011.

[13]GS1 EPCglobal, Regulatory status for using RFID in the EPC Gen 2 band (860 to $960 \mathrm{MHz}$ ) of the UHF spectrum, 31 October 2014.

[14]S. J. Orfanidis, Electromagnetic Waves and Antennas, Rutgers University, 2000.

[15] J. D. Jackson, Classical Electrodynamics, Wiley, 1998.

[16]D. Pozar, New results for minimum Q, maximum gain, and polarization properties of electrically small arbitrary antennas, IEEE EuCAP, 2009.

[17]P. N. Murgatroyd, Calculation of proximity losses in multistranded conductor bunches, IEEE Proc. A (Phys. Science, Meas. Inst., Manag.and Educ.), vol. 136, issue 3, pp. 115-120, May 1989.

[18] K. V. S. Rao, P. V. Nikitin, S. F. Lam, Antenna Design for UHF RFID Tags: A Review and a Practical Application, IEEE Trans. Antennas Prop., vol. 53, (12), pp. 3870-3876, December 2005. 